\title{
BMJ Open Quality Improving the quality of physical health monitoring in CAMHS for children and adolescents prescribed medication for ADHD
}

\author{
Cristal Oxley, Omer S Moghraby, Rani Samuel, Dan W Joyce
}

To cite: Oxley C, Moghraby OS, Samuel R, et al. Improving the quality of physical health monitoring in CAMHS for children and adolescents prescribed medication for ADHD.BMJ Open Quality 2018;7:e000213. doi:10.1136/ bmjoq-2017-000213

Received 22 September 2017 Accepted 5 April 2018
Check for updates

Department of Child and Adolescent Psychiatry, South London and Maudsley NHS Foundation Trust, London, UK

Correspondence to

Dr Cristal Oxley;

cristal.oxley@slam.nhs.uk

\section{ABSTRACT}

Attention deficit hyperactivity disorder (ADHD) is a common neurodevelopmental disorder characterised by a persistent, pervasive pattern of inattention, impulsivity and hyperactivity. Stimulant medication such as methylphenidate has an established evidence base in the treatment of children and adolescents with ADHD. However, it is also associated with a risk of side effects which may include decreased appetite, increased blood pressure and possible reduced growth. Monitoring physical health in children and adolescents prescribed medication for ADHD is a key clinical responsibility and includes a number of parameters as outlined in the National Institute for Health and Care Excellence Guidelines. Ascertaining the centiles of physical observations is essential to put these into developmental context and accurately inform treatment decisions. This quality improvement project aimed to improve physical health monitoring in children and adolescents prescribed stimulant medication for ADHD within a large specialist urban inner-city Child and Adolescent Mental Health Service (CAMHS) in South London and Maudsley NHS Foundation Trust. Baseline data were obtained to establish the quality of physical monitoring including blood pressure, height, weight and centiles. Targeted interventions included the development of a novel web-based application designed to calculate and record centiles. We report an improvement in total proportion compliance with physical health monitoring from $24 \%$ to $75 \%$. The frequency of recording baseline blood pressure centiles increased from $0 \%$ to $62 \%$; recording baseline height centiles increased from $37 \%$ to $81 \%$ and recording baseline weight centiles increased from $37 \%$ to $81 \%$. Improvement in the delivery of highquality care was achieved and sustained through close collaboration with clinicians involved in the treatment pathway in order to elicit and respond effectively to feedback for improvement and codevelop interventions which were highly effective within the clinical system. We believe this model to be replicable in other CAMHS services and ADHD clinics to improve the delivery of highquality clinical care.

\section{PROBLEM}

Assessment and monitoring of the physical health of children and adolescents prescribed stimulant medication for attention deficit hyperactivity disorder (ADHD) is a key clinical responsibility as outlined in the National Institute for Health and Care Excellence (NICE) Guidelines CG72. ${ }^{1}$ Physical examination including the centiles of observations should be documented in the patient's medical record. Traditionally, growth charts have been used to calculate and record centiles. Increasingly, however, patient medical records are electronic and this requires a change in the way in which clinical information is documented. We observed that the clinical process involved a friction in terms of the requirement to look up centiles on a paper growth chart, an intrinsically time-consuming and error-prone task, and then a duplication in workload in the requirement to then type these into the medical record. We identified that documentation of physical assessment was poor and inconsistent at baseline. We were interested in the development of efficient, reliable and sustainable solutions to this problem.

We aimed to improve the proportion compliance with recording centiles of observations (blood pressure, height and weight) at commencement of stimulant medication to $75 \%$ over a 12 -month period.

\section{BACKGROUND}

ADHD is one of the most common neurodevelopmental disorders of childhood. In the UK, a survey of 10438 children and adolescents aged between 5 and 15 years found that $3.62 \%$ of boys and $0.85 \%$ of girls had ADHD. ${ }^{2}$ Stimulant medication such as methylphenidate is the most frequently prescribed medication for the treatment of ADHD in children and adolescents in the UK. Stimulant medication acts at the central nervous system and is associated with a risk of side effects which may include reduced appetite and weight, ${ }^{3}$ increased blood pressure ${ }^{45}$ and pulse rate, ${ }^{3}$ and possible reduced growth. ${ }^{6-8}$ UK NICE Guidelines $^{1}$ and European Guidelines ${ }^{9} 10$ 
recommend that children and adolescents should have a comprehensive baseline physical assessment including height, weight and blood pressure and that these should be plotted on standardised growth charts and monitored regularly during treatment. The authors are not aware of any studies or initiatives to support clinicians in meeting these requirements using an electronic platform.

\section{BASELINE MEASUREMENT}

Baseline data were collected in a randomly selected sample of 50 cases. Cases were identified as children and adolescents who were issued a new prescription to commence stimulant medication for ADHD during the 10-year period following the introduction of electronic medical records in the service. Nine cases were subsequently excluded since they did not meet study criteria. The main reason for exclusion was that stimulant medication was subsequently not commenced. A pro forma was used to record whether components of baseline physical assessment were completed.

The primary outcome measure is the proportion compliance with baseline documentation of centiles for blood pressure, height and weight. For instance, 100\% proportion compliance represents the documentation of all three centiles at baseline in one case during one clinical encounter.

Secondary outcome measures include other components of the baseline physical assessment as recommended in NICE Guidelines including documentation of blood pressure, pulse, height, weight, medical history, family history and ECG if indicated. The total proportion compliance with NICE Guidelines recommended baseline physical assessment represents a composite statistic for the secondary outcome measures.

In the baseline sample, the proportion compliance with recording centiles was $24 \%$. In more detail, blood pressure centiles were documented in $0 \%(n=0)$ of cases and height and weight centiles were each documented in $37 \%$ of cases $(n=15)$. With regard to secondary outcome measures, the median total proportion compliance with all components of baseline physical assessment as recommended by NICE Guidelines at baseline was $58 \%$. In order to establish whether a change in the quality of physical monitoring occurred, we planned to continue to measure the process using the same pro forma following the introduction of tailored interventions.

\section{DESIGN}

The multidisciplinary project team consisted of two consultant child and adolescent psychiatrists, two specialist trainees in child and adolescent psychiatry, four core trainees in psychiatry, a clinical psychologist, clinical nurse prescriber and a foundation year 1 doctor. Following close discussion with the team, we agreed that in order to best support clinicians to make positive changes in the quality of physical health monitoring, we needed to improve the process of calculation and documentation of centiles to improve reliability and efficiency. A novel web-based application (app) was developed to calculate the centiles of physical observations. The app could also be used to facilitate recording of observations where a free text copy-paste approach was included so data could be pasted directly into the patient's electronic medical record. The app was made freely available on all clinicians' mobile devices and computers. All ADHD prescribers in the service were trained to use the tool.

During the course of the project, several new clinicians joined the organisation including trainees rotating through the department. An orientation regarding the tool was included in induction. Clinicians using the tool met on a monthly basis and feedback was obtained.

\section{STRATEGY}

Our aim was to improve the quality of baseline physical assessment of children and adolescents prescribed stimulant medication for ADHD by improving the proportion compliance with recording centiles (blood pressure, height and weight) to $75 \%$ over a 12 -month period. We undertook two Plan-Do-Study-Act (PDSA) cycles.

\section{PDSA cycle 1}

The web-based app was introduced to all prescribers across three clinical teams and training and technical support was provided. Clinicians were asked to use this tool for all new ADHD assessments. This initial intervention led to an increase in the proportion compliance in recording centiles to $81 \%$. The clinical team fed back that the tool was easy to use, effective and reliable. It was suggested that the process could be streamlined further by embedding the tool directly in the electronic medical records. The lead psychiatrist advised us that several new clinicians were joining the service with no prior experience of the tool.

\section{PDSA cycle 2}

A series of training and problem-solving sessions were held to support clinicians maximise the utility of the tool including inductions for clinicians joining the service. The tool was made available on mobile devices and computers for all clinicians to improve access. We liaised with the trust Information and Communications Technology department regarding embedding the tool into the electronic medical record system. Following this cycle, proportion compliance in recording centiles was $75 \%$.

\section{RESULTS}

The primary outcome measure was the proportion compliance with documentation of baseline centiles for blood pressure, height and weight. Data were measured continuously over the 12-month period and proportion compliance plotted against time (figure 1 ). The proportion compliance improved from $24 \%$ at baseline to $75 \%$ after 12 months. A marked initial increase to $81 \%$ 


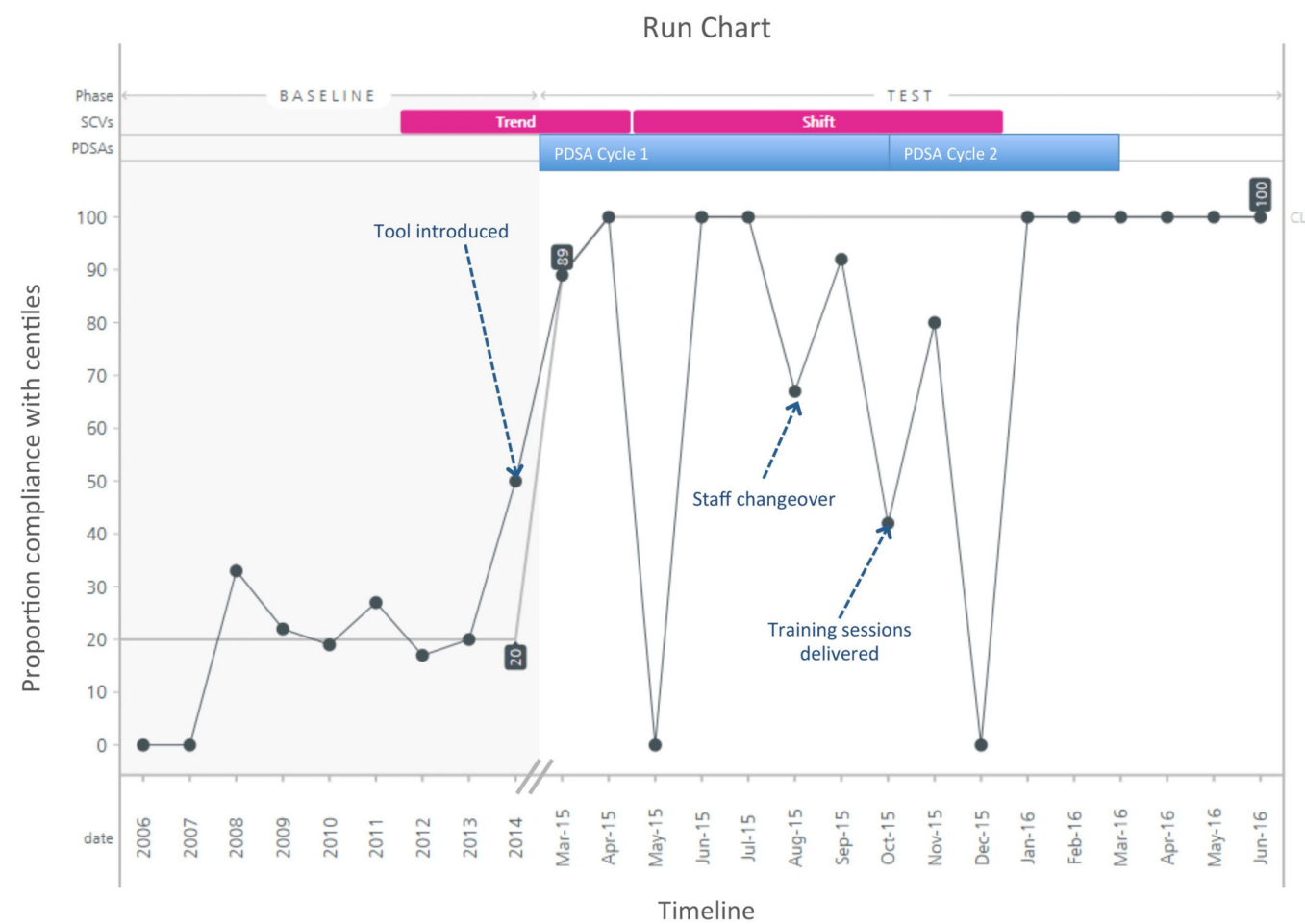

Figure 1 Run chart of the proportion compliance of recording centiles of physical observations against time. PDSA, Plan-DoStudy-Act; SCV, special cause variation.

proportion compliance was observed following PDSA cycle 1. Following PDSA cycle 2, a proportion compliance of $75 \%$ was observed. In terms of the trend, the final 6 months of the study represent consistent and sustained achievement of $100 \%$ proportion compliance in all clinical cases.

With regard to secondary outcome measures, the median total proportion compliance with NICE Guidelines recommended baseline physical assessment increased from $58 \%$ to $83 \%$ over the 12-month period.

A degree of fluctuation in proportion compliance can be seen in the run chart during both PDSA cycles. A temporal association was observed with several events and interventions including the introduction of the tool, changes in clinical staff and additional training.

\section{LESSONS AND LIMITATIONS}

The project aim was to improve the quality of physical assessment in children and adolescents prescribed stimulant medication for ADHD. In order to achieve this we liaised closely with the clinical team and developed a tailored intervention which was helpful, reliable and efficient to use in the clinical environment. We also introduced a framework to support clinicians using the tool. We found that listening closely to the needs of clinicians with regular opportunities for feedback was crucial in improving quality of care in a practical and sustainable manner within this busy service. In doing so, the tool has become well established in the clinical setting and treatment pathways and systems to monitor physical health have been integrated in the development of a new ADHD clinic. The clinical impact of this project was wider than initially anticipated, representing a range of meaningful improvements in quality of care. For example, prompt identification of elevated blood pressure centiles led to conversations with families with regard to modifiable factors including smoking, diet and exercise while also informing prescribing decisions. Families became better engaged in treatment and, in several cases, features of comorbid conduct disorder also reduced following improved levels of engagement with treatment generally including parenting interventions. Similarly, by engaging with lifestyle recommendations, improvements in blood pressure centiles were also observed. A limitation of this study is that it was not designed to prove causation nor do we make claims to this effect.

Overall a substantial improvement in the quality of physical assessment was observed. However, a notable degree of fluctuation can be seen in the data, particularly during PDSA cycle 2 . This is related in part to the natural variation in frequency of assessments according to clinical demand and the resulting degree of influence of each individual case on the mean given the relatively small sample size $(n=33)$ which represents a limitation of this project. Feedback from the multidisciplinary team revealed that variability may also represent practical barriers to implementing change in a consistent manner across the service. For example, varied availability of resources such as limited computer access in clinic rooms can present barriers to utilising an electronic platform. The follow-up period spans periods of change-over in clinical staff. We cannot exclude possible confounding bias. 
We attempted to minimise the impact of staff change-over on quality by training colleagues on the tool early and offering technical support as required.

There are several potential barriers in the development and implementation of informatics and software in healthcare systems. By using a pragmatic open source approach in the development of this web-based app we were able to create and use the technology in clinical practice promptly. We did, however, then face the challenge of how best to incorporate the tool directly into the electronic medical record system. Although this was identified early, there were several technical barriers to implementing this within the time frame of this project including interfacing with the current electronic record system. There is now a wider project within the trust to further develop and integrate these systems.

\section{CONCLUSION}

This quality improvement project set out to improve the quality of physical health assessment in children and adolescents with ADHD. We have achieved this aim with an improvement in proportion compliance in recording centiles from $24 \%$ to $75 \%$ over 12 months. This represents a meaningful improvement in quality of care across the clinical service. Clinical staff report additional benefits including an improved understanding of monitoring requirements while also saving time through improved efficiency in systems.

The 12-month follow-up period has proven sufficient to demonstrate that the improvement in proportion compliance has been sustained at or above this level. The proportion compliance in documenting centiles reaches $100 \%$ compliance which is sustained during the last 6 months, as can be seen on the run chart.

In view of the significant improvement in quality of clinical practice associated with this unique intervention, we are in the process of disseminating findings within our NHS trust and also to prescribers in the wider network. We are engaged in discussion within our trust with regards to formally incorporating this tool into the electronic medical record to further improve quality, usability and sustainability. Readers may access the code for the app which has been made freely available via the following link: https://github.com/danwjoyce/centilesshiny-app.git

Acknowledgements The authors thank the Lewisham CAMHS service including Dr Penelope Lowe, Miss Jessica Brunet and Dr John Ayorinde for their valuable contributions to this project.
Contributors RS, OSM and DJ were involved in the design of the project. DJ developed the web-based application. CO and DJ collected and analysed the data together with colleagues from the clinical service. The article was drafted by $\mathrm{CO}$ and revised by CO, RS, OSM and DJ. The final version was approved by CO, RS, OSM and DJ.

Funding The authors have not declared a specific grant for this research from any funding agency in the public, commercial or not-for-profit sectors.

Competing interests None declared.

Patient consent Not required.

Ethics approval According to the policy at South London and Maudsley NHS Foundation Trust, this work meets criteria for quality improvement activities exempt from ethics review.

Provenance and peer review Not commissioned; externally peer reviewed.

Open Access This is an Open Access article distributed in accordance with the Creative Commons Attribution Non Commercial (CC BY-NC 4.0) license, which permits others to distribute, remix, adapt, build upon this work non-commercially, and license their derivative works on different terms, provided the original work is properly cited and the use is non-commercial. See: http://creativecommons.org/ licenses/by-nc/4.0/

(c) Published by the BMJ Publishing Group Limited. For permission to use (where not already granted under a licence) please go to http://www.bmj.com/company/ products-services/rights-and-licensing/

\section{REFERENCES}

1. National Institute for Health and Clinical Excellence. Diagnosis and management of $A D H D$ in children, young people and adults. National Clinical Practice Guideline Number 72. London: NICE, 2008.

2. Ford T, Goodman R, Meltzer H. The British Child and Adolescent Mental Health Survey 1999: the prevalence of DSM-IV disorders. J Am Acad Child Adolesc Psychiatry 2003;42:1203-11.

3. Storebø OJ, Ramstad E, Krogh HB, et al. Methylphenidate for children and adolescents with attention deficit hyperactivity disorder (ADHD). Cochrane Database Syst Rev 2015;25:CD009885.

4. Hammerness PG, Perrin JM, Shelley-Abrahamson R, et al. Cardiovascular risk of stimulant treatment in pediatric attention-deficit/hyperactivity disorder: update and clinical recommendations. J Am Acad Child Adoles Psychiatry 2011;50:978-90.

5. Hennissen L, Bakker MJ, Banaschewski T, et al. Cardiovascular Effects of Stimulant and Non-Stimulant Medication for Children and Adolescents with ADHD: a systematic review and meta-analysis of trials of Methylphenidate, Amphetamines and Atomoxetine. CNS Drugs 2017;31:199-215.

6. Faraone SV, Biederman J, Morley CP, et al. Effect of stimulants on height and weight: a review of the literature. J Am Acad Child Adolesc Psychiatry 2008;47:994-1009.

7. Poulton A. Growth on stimulant medication; clarifying the confusion: a review. Arch Dis Child 2005;90:801-6.

8. Swanson J, Arnold LE, Kraemer H, et al. Evidence, interpretation, and qualification from multiple reports of long-term outcomes in the Multimodal Treatment study of Children With ADHD (MTA): part I: executive summary. J Atten Disord 2008;12:4-14.

9. Graham J, Banaschewski T, Buitelaar J, et al. European guidelines on managing adverse effects of medication for ADHD. Eur Child Adoles Psychiatry 2011;20:17-37.

10. Taylor E, Döpfner M, Sergeant J, et al. European clinical guidelines for hyperkinetic disorder - first upgrade. Eur Child Adolesc Psychiatry 2004;13:17-30. 